\title{
DEGREES OF UNSOLVABILITY OF FIRST ORDER DECISION PROBLEMS FOR FINITELY PRESENTED GROUPS
}

\author{
OLEG V. BELEGRADEK \\ (Communicated by Andreas R. Blass)
}

\begin{abstract}
We show that for any arithmetical $m$-degree $\mathbf{d}$ there is a first order decision problem $\mathbf{P}$ such that $\mathbf{P}$ has $m$-degree $\mathbf{d}$ for the free 2-step nilpotent group of rank 2. This implies a conjecture of Sacerdote.
\end{abstract}

Let $\varphi(\mathbf{v})$ be a formula of the first order language of group theory $L$ and $\pi$ a finite group presentation in an alphabet $X$. The first order decision problem $(? \mathbf{v}) \varphi(\mathbf{v})$ for $G_{\pi}$, the group defined by $\pi$, is the problem of deciding for arbitrary tuple of words $\mathbf{u}$ in $X$ whether or not the sentence $\varphi(\mathbf{u})$ holds in $G_{\pi}$. For example, $(? v) v=1$ is the word problem and $(? x y)(\exists z) x^{z}=y$ is the conjugacy problem. It is easy to see that for a finitely presented group $G$ the type of recursive isomorphism of the problem $(? \mathbf{v}) \varphi(\mathbf{v})$ is independent of which finite presentation of $G$ one chooses.

Sacerdote [S1] posed the following problem: which Turing degrees of unsolvability are the degrees of first order decision problems for finitely presented groups? For any atomic formula $\varphi(\mathbf{v})$ the problem $(? \mathbf{v}) \varphi(\mathbf{v})$ is recursively enumerable; therefore any first order decision problem for any finitely presented group is arithmetical. Boone suggested that all first order decision problems for finitely presented groups have recursively enumerable Turing degrees. Sacerdote [S2] showed that it is not so. He constructed an ad hoc rather complicated example of a finitely presented group $G$ and a formula $\varphi(\mathbf{v})$ with four free variables such that the problem $(? \mathbf{v}) \varphi(\mathbf{v})$ has degree $\mathbf{0}^{\prime \prime}$ for $G$. He suggested the following

Conjecture. Let $\mathbf{D}$ be an arithmetical degree of unsolvability. Then there exist a first order decision problem $\mathbf{P}$ and a finitely presented group $G$ such that $\mathbf{P}$ has degree $\mathbf{D}$ for $G$.

The aim of the present note is to prove this conjecture in a stronger form.

Theorem. Let $F$ be the free 2-step nilpotent group of rank 2. Then for any arithmetical $m$-degree $\mathbf{d}$ there is an L-formula $\varphi_{\mathbf{d}}(v)$ with one free variable such that the problem $(? v) \varphi_{\mathbf{d}}(v)$ has $m$-degree $\mathbf{d}$ for $F$.

Note that $F$ is finitely presented: if $a_{1}$ and $a_{2}$ freely generate $F$, then the relations $\left[a_{1}, a_{2}, a_{1}\right]=1$ and $\left[a_{1}, a_{2}, a_{2}\right]=1$ define $F$.

Received by the editors August 19, 1994.

1991 Mathematics Subject Classification. Primary 03D40, 03D30, 20F10, 20F18.

Key words and phrases. First order decision problem, $m$-degree.

The author was partially supported by the AMS fSU Aid Fund. 
For a proof of the theorem we need some known facts.

(A) Every group word in $a_{1}, a_{2}$ can be effectively reduced in $F$ to the form $a_{2}^{k} a_{1}^{l} c^{m}$, where $c=\left[a_{1}, a_{2}\right]$; the presentation of any element in this form is unique.

(B) A pair of elements $\left(h_{1}, h_{2}\right)$ is said to be a base in a group $H$ if

(1) $H$ is 2-step nilpotent,

(2) $C_{H}\left(h_{1}\right)$ and $C_{H}\left(h_{2}\right)$ are abelian,

(3) $C_{H}\left(h_{1}, h_{2}\right)=Z(H)$,

(4) $\left[C_{H}\left(h_{1}\right), h_{2}\right]=\left[h_{1}, C_{H}\left(h_{2}\right)\right]=Z(H)$.

Note that there is an $L$-formula $\beta\left(x_{1}, x_{2}\right)$ such that, for any group $H$ and its elements $h_{1}$ and $h_{2}$, the pair $\left(h_{1}, h_{2}\right)$ is a base in $H$ iff $\beta\left(h_{1}, h_{2}\right)$ holds in $H$.

The following construction is due to Mal'tsev $[\mathbf{M}]$. Let $\left(h_{1}, h_{2}\right)$ be a base in $H$. Define binary operations + and $\times$ on $Z(H)$ as follows. For $z_{1}, z_{2}$ in $Z(H), z_{1}+z_{2}$ is just $z_{1} z_{2}$, and $z_{1} \times z_{2}$ is defined to be $\left[g_{1}, g_{2}\right]$, where $g_{i} \in C_{H}\left(h_{i}\right)$ for $i=1,2$, $\left[g_{1}, h_{2}\right]=z_{1},\left[h_{1}, g_{2}\right]=z_{2}$. It turns out that $\times$ is well defined and $(Z(H),+, \times)$ is a ring with identity; denote the ring by $\operatorname{Ring}\left(H, h_{1}, h_{2}\right)$. Clearly, the ring is definable in $H$ with parameters $h_{1}, h_{2}$. Therefore for any formula $\psi(\mathbf{v})$ of $\mathcal{L}$, the first order language of ring theory, one can effectively construct an $L$-formula $\psi^{*}\left(\mathbf{v}, x_{1}, x_{2}\right)$ such that, for any group $H$ with a base $\left(h_{1}, h_{2}\right)$ and any tuple $\mathbf{z}$ in $Z(H)$, the formula $\psi(\mathbf{z})$ holds in $\operatorname{Ring}\left(H, h_{1}, h_{2}\right)$ iff the formula $\psi^{*}\left(\mathbf{z}, h_{1}, h_{2}\right)$ holds in $H$.

$\mathrm{Mal}^{\prime}$ tsev showed that $\left(a_{1}, a_{2}\right)$ is a base in $F$; the $\operatorname{ring} \operatorname{Ring}\left(F, a_{1}, a_{2}\right)$ consists of elements of the form $c^{n}$ and is isomorphic to $\mathbf{Z}$ via $c^{n} \leftrightarrow n$.

(C) In $F$ any two bases are conjugate by an automorphism [B, Theorem 5.11], even though in general it is not the case. So $b_{1}, b_{2}$ freely generate $F$ iff $\beta\left(b_{1}, b_{2}\right)$ holds in $F$.

Proof. If $\mathbf{d}$ is the $m$-degree of the set of all natural numbers $N$ we can take $v=v$ as $\varphi_{\mathbf{d}}(v)$. Suppose $\mathbf{d}$ is the $m$-degree of a proper arithmetical subset of $N$. Choose $A \varsubsetneqq \mathbf{Z}$ of $m$-degree $\mathbf{d}$ such that $n \in A$ iff $-n \in A$, for any integer $n$. Due to the arithmeticity of $A$, there is an $\mathcal{L}$-formula $\psi(v)$ defining $A$ in the ring $\mathbf{Z}$. We show that the $L$-formula $\left(\exists x_{1} x_{2}\right)\left(\beta\left(x_{1}, x_{2}\right) \wedge \psi^{*}\left(v, x_{1}, x_{2}\right)\right)$ can be taken as $\varphi_{\mathbf{d}}(v)$.

First we note that $n \in A$ iff $\varphi_{\mathbf{d}}\left(c^{n}\right)$ holds in $F$. Suppose $n \in A$. Then $\psi(n)$ holds in $\mathbf{Z}$. Therefore $\psi\left(c^{n}\right)$ holds in $\operatorname{Ring}\left(F, a_{1}, a_{2}\right)$, that is, $\psi^{*}\left(c^{n}, a_{1}, a_{2}\right)$ holds in $F$. So we can take $a_{1}, a_{2}$ as $x_{1}, x_{2}$. Now suppose that $\varphi_{\mathbf{d}}\left(c^{n}\right)$ holds in $F$. Then there are $b_{1}, b_{2}$ in $F$ such that $\beta\left(b_{1}, b_{2}\right)$ and $\psi^{*}\left(c^{n}, b_{1}, b_{2}\right)$ hold in $F$. Because of (C), there is an automorphism $\tau$ of $F$ sending $b_{i}$ to $a_{i}$, for $i=1,2$. Then $\tau(c)^{n}$ satisfies $\psi^{*}\left(v, a_{1}, a_{2}\right)$ in $F$. The center of $F$ is the infinite cyclic group generated by $c$. As $\tau(c)$ also generates the center, $\tau(c)$ is $c$ or $c^{-1}$. Therefore $\psi\left(c^{n}\right)$ or $\psi\left(c^{-n}\right)$ holds in $\operatorname{Ring}\left(F, a_{1}, a_{2}\right)$. So $\psi(n)$ or $\psi(-n)$ holds in $\mathbf{Z}$, that is, $n \in A$ or $-n \in A$. Then $n \in A$, by the choice of $A$.

Now it is clear that $A$ is 1-reducible to the problem $(? v) \varphi_{\mathbf{d}}(v)$ for $F$. Fix an integer $m_{0}$ outside $A$. For a word $w$ in $a_{1}, a_{2}$ put

$$
g(w)= \begin{cases}m_{0} & \text { if } \quad w=a_{2}^{k} a_{1}^{l} c^{m} \text { in } F \text { and } k \neq 0 \text { or } l \neq 0 \\ m & \text { if } \quad w=c^{m} \text { in } F .\end{cases}
$$

Clearly, $g$ is a recursive function, and $\varphi_{\mathbf{d}}(w)$ holds in $F$ iff $g(w) \in A$. So the problem $(? v) \varphi_{\mathbf{d}}(v)$ for $F$ is $m$-reducible to the set $A$. 


\section{REFERENCES}

[B] O. V. Belegradek, The Mal'cev correspondence revisited, Proc. Int. Conf. on Algebra Dedicated to the Memory of A. I. Mal'cev (L. A. Bokut', Yu. L. Ershov, and A. I. Kostrikin, eds.), Contemp. Math., vol. 131, Amer. Math. Soc., Providence, RI, 1992, pp. 37-59. MR 93m:03059

[M] A. I. Mal'tsev, A correspondence between rings and groups, Mat. Sb. (N.S.) 50 (1960), 257266; English transl., The metamathematics of algebraic systems. Collected papers: 19361967, North-Holland, 1971, pp. 124-137.

[S1] G. S. Sacerdote, Some unsolvable decision problems in group theory, Proc. Amer. Math. Soc. 36 (1972), 231-238. MR 47:8660

[S2] _ _ On a problem of Boone, Math. Scand. 31 (1972), 111-117. MR 47:6871

Kemerovo State University, Kemerovo 650043, Russia

E-mail address: beleg@kaskad.kemerovo.su 